\title{
Human immune diversity: from evolution to modernity
}

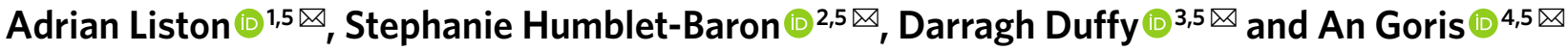

The extreme diversity of the human immune system, forged and maintained throughout evolutionary history, provides a potent defense against opportunistic pathogens. At the same time, this immune variation is the substrate upon which a plethora of immune-associated diseases develop. Genetic analysis suggests that thousands of individually weak loci together drive up to half of the observed immune variation. Intense selection maintains this genetic diversity, even selecting for the introgressed Neanderthal or Denisovan alleles that have reintroduced variation lost during the out-of-Africa migration. Variations in age, sex, diet, environmental exposure, and microbiome each potentially explain the residual variation, with proof-of-concept studies demonstrating both plausible mechanisms and correlative associations. The confounding interaction of many of these variables currently makes it difficult to assign definitive contributions. Here, we review the current state of play in the field, identify the key unknowns in the causality of immune variation, and identify the multidisciplinary pathways toward an improved understanding.

$\mathrm{T}$ he immune system possesses immense individual-toindividual diversity. Immunity is intrinsically variable, because it is controlled by the most polymorphic genes and is shaped by highly sensitive environmental sensors that are capable of pushing immunity into myriad functional configurations. These functional configurations are the intrinsic biases toward particular immune response types. Thus, while most healthy humans have the capacity to turn on type 1 helper $T$ cells ( $\mathrm{T}_{\mathrm{H}} 1$ cells), $\mathrm{T}_{\mathrm{H}} 2$ cells, $\mathrm{T}_{\mathrm{H}} 17$ cells, type I interferons, inflammasome activation, and a multitude of other states, individuals differ in the degree to which they are primed for each functional configuration ${ }^{1-3}$. These interindividual differences are relatively low at birth ${ }^{4}$ but continually expand as we age and encounter new environments ${ }^{1,5}$, and are both stable and robust to perturbation ${ }^{2,3}$. The origin of this diversity is rooted in our evolutionary pasts, with genes that control immune traits being among the most divergent in archaic genomes ${ }^{6}$. In modern humans, a diverse range of immune-associated disorders reflects the clinical consequences of this diversity in immunological states. This diversity also provides challenges for more successful application of immunotherapeutic strategies, initially developed against cancer or autoimmunity, that hold wider potential for other clinical conditions. For example, despite their widespread success in successfully treating 21 types of cancer, checkpoint blockade inhibitors induce adverse immune effects in up to $50 \%$ of people who receive combination therapy ${ }^{7}$. A better understanding of the reasons driving such variability could help define more precisionmedicine strategies.

The immune system is possibly unique in the advantages that variation can confer. The Red Queen hypothesis, an evolutionary arms race between competing species (Fig. 1a), runs into a generational time asymmetry when considering the evolution of pathogens and hosts (Fig. 1b). Rather than unsustainable convergence toward a homogenous state of infection-resistance, evolution has selected for maintenance of immune diversity as a protective mechanism
(Fig. 1c). When potential pathogens can rapidly specialize to take advantage of a fixed niche, an evolutionary advantage can be gained from possessing an immune system wired into a functional configuration that is different from that of the prior host (Fig. 1c,d). Beyond the immune system, a single holotype can be considered optimal per environmental condition, with diversity representing a divergence from the optimum. In the immune system, by contrast, diversity is generally a beneficial feature (although some loci, such as the Toll-like receptor (TLR) loci, are subject to intense purifying selection $^{8}$ ), with increased immune divergence from the prior host predicted to benefit the newly infected individual.

In this Review, we outline the current state of knowledge on the primary drivers of immunological variation. We concentrate on serological, cellular, and molecular aspects of immunity, as direct measures of immune variation, instead of clinical outcomes and other complex phenotypes, of which immune status is only one aspect. Likewise, this Review focuses on the drivers of immune variation that have a substantial impact at the population level common genetic variants, intrinsic factors such as age and sex, and common environmental exposures. Of necessity, we omit rare drivers, such as rare genetic variants, except for the lessons they provide for a broader population-level understanding.

\section{Genetic drivers of immune variation}

Genetic variation is an important driver of immune variation. Studies of multigenerational families ${ }^{9,10}$ and twin pairs ${ }^{11-13}$ nevertheless highlight high variability in how much genetic variation contributes to different immune traits. The largest multigenerational study $^{10}$ demonstrates a median heritability of $37 \%$ for immune variation, with a range between $0 \%$ and $79 \%$, in line with most previous studies. Cytokine pathways, key drivers of immunity, are particularly heritable ${ }^{12,14,15}$. Genome-wide association studies (GWASs) allow the dissection of this aggregate genetic contribution to individual causal drivers.

'Immunology Programme, The Babraham Institute, Babraham Research Campus, Cambridge, United Kingdom. ${ }^{2}$ KU Leuven, University of Leuven, Department of Microbiology, Immunology and Transplantation, Leuven, Belgium. ${ }^{3}$ Translational Immunology Lab, Department of Immunology, Institut Pasteur, Paris, France. ${ }^{4}$ KU Leuven, University of Leuven, Leuven Brain Institute, Department of Neurosciences, Leuven, Belgium. ${ }^{5}$ These authors contributed equally: Adrian Liston, Stephanie Humblet-Baron, Darragh Duffy, An Goris. ${ }^{凶}$-mail: adrian.liston@babraham.ac.uk; 


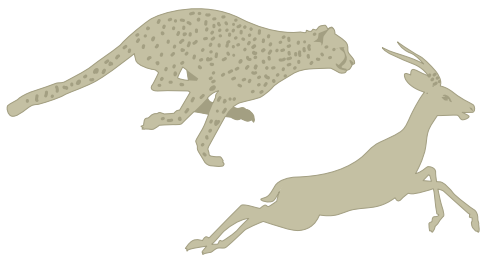

b

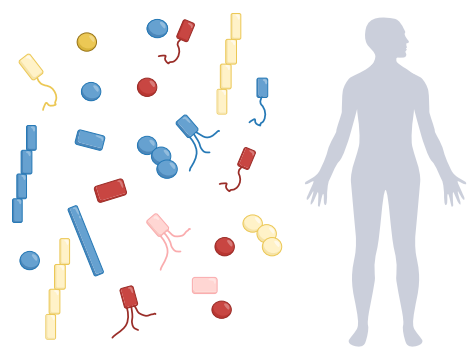

c



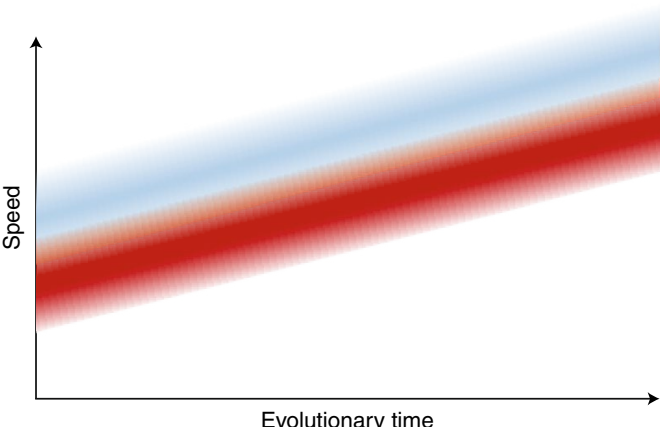

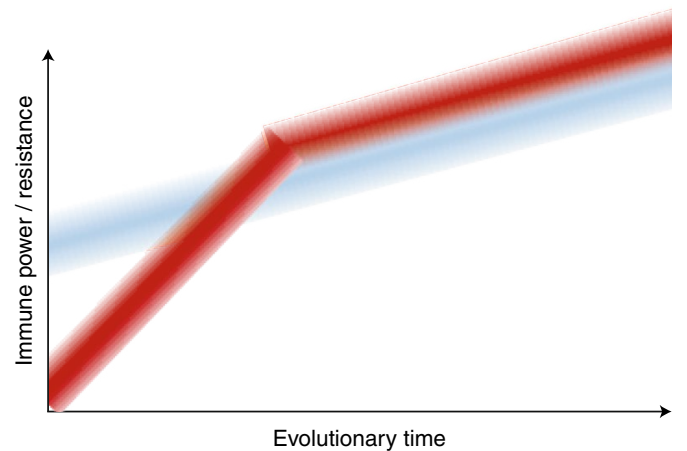

d

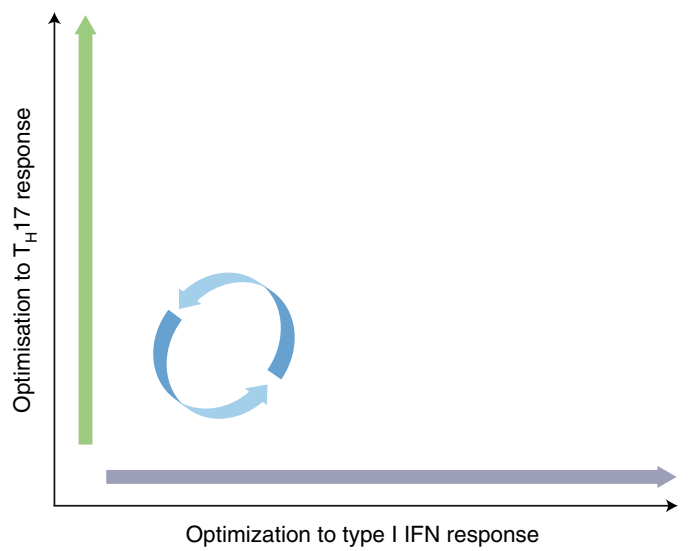

Fig. 1 | Immunological diversity as an evolutionary strategy. a, In a simplified single-trait Red Queen scenario, a predator-prey relationship based on speed drives ever-increasing speed in both the predator (red density curve) and prey (blue density curve), with the fast end of the predator bell curve requiring an overlap with the slow end of the prey bell curve. $\mathbf{b}$, In a host-pathogen relationship, the asymmetry in population growth potential and generation time would allow the pathogen to rapidly specialize to counter any single-trait immune response. c, Representation of a human population with immune diversity, including individuals biased toward different immune responses. Individual $X$ is immunologically more similar to individual $Y$ than they are to individual $Z$. These distances also relate to the magnitude of the reoptimization cost that a pathogen will experience on moving from host $X$ to either host $Y$ or host $Z$. d, Evolutionary trajectories of pathogens in their optimization toward two example immune biases. The green trajectory represents optimization in a host population in which all individuals are biased toward $\mathrm{T}_{H} 17$. The purple trajectory represents optimization in a host population in which all individuals are biased toward type I interferons (IFN). The blue trajectory represents optimization in an immunologically diverse host population.

Extremely large GWASs have been performed to investigate basic hematological traits, and have identified $>7,000$ loci associated with such traits ${ }^{16,17}$. Colocalization of immune-modifying variants with risk variants for autoimmune diseases has been observed, suggesting clinical consequences ${ }^{16}$. These very large studies permit trans-ethnic analysis. For example, a missense variant in IL7 is associated with increased lymphocyte counts in South Asians, an effect that has been obscured in other populations ${ }^{17}$. The other end of the GWAS spectrum trades power for depth: six fine-detail immune-phenotyping GWASs have been performed on relatively small numbers of individuals ${ }^{9-11,18-20}$. Together, these GWASs have identified 95 loci associated with immune phenotypes, of which $28 \%$ have been reported by more than one study. Factors to consider when comparing studies are the design of the immune-phenotyping platform, that power is still limited, and differences in ancestry. For example, $13 \%$ of associations in a Sardinian population ${ }^{10}$ have a twofold-higher frequency in this population than in the European cohort of the 1000 Genomes Project, where they are mostly at low frequency. Hence, more associations are expected to emerge and, as for hematological traits, analyses of different ancestries will be helpful.

The wealth of genetic data allows broad comparisons to be made. Associations have been predominantly with protein quantitative trait loci (pQTLs), rather than cellular traits. These pQTLs act mostly in trans, via regulatory genes ${ }^{19,21-23}$. This is in line with distal (trans) effects being an important contributor to variation of gene 
expression among immune cell types and being enriched for disease associations, even though most expression QTL (eQTL) studies so far have focused on cis effects ${ }^{24}$. Increasingly, physiological perturbations in specific cell types are being mapped and are assisting in the evaluation of the colocalization of immune trait GWASs and eQTL signals ${ }^{25}$. More recently, splice variation has been identified as an additional source of variation that is increased following immune activation ${ }^{26}$. Genetic determinants of alternative splicing are largely independent of those of gene regulation and are often context dependent or alter splicing only in the context of immune stimulation ${ }^{26}$.

The human leukocyte antigen (HLA) locus, the most genetically diverse in the human genome, maintained by balancing selection ${ }^{27}$, is worth additional attention. This locus has been reproducibly associated with multiple immunological disorders, both infectious and autoimmune, often with the strongest effect size $\mathrm{e}^{28}$. These associations may be driven by variation in permission of clonal HLApeptide-TCR/KIR interactions; however, a role for variation in HLA expression level and stability is being increasingly inferred as a peptide-independent basis for association ${ }^{28}$. GWASs for healthy, baseline immune phenotypes have revealed several independent associations within the HLA locus, including a known type 1 diabetes $H L A-D R B 1$ risk allele that impacts HLA-DRB1 surface expression in innate immune cells ${ }^{20}$, and variants correlated with surface expression of costimulatory molecules in adaptive immune cells ${ }^{10}$. HLA has an even more prominent role in the genetic control of immunoglobulin levels, accounting for $19 \%$ of known associations and including autoimmune disease-associated alleles ${ }^{29}$. On the basis of the biology of HLA variation, it is expected that future GWASs of immune responses at the clonal level will identify stronger effects, accounting for the powerful associations exhibited with disease.

\section{Age and immune variation}

Age is one of the most potent drivers of immune variation, driving a shift toward systemic inflammation and away from naive lymphocyte phenotypes ${ }^{1,3,5}$. Age correlates with multiple immunological parameters and also amplifies the degree of variation present, a result of direct effects of age as well as increased environmental exposures over time ${ }^{1,12}$. Age-related immune variation can be due to cumulative effects of immunosenescence, such as lower activity of hematopoietic stem cells ${ }^{30,31}$, altered lineage differentiation ${ }^{32}$, thymic involution $^{33}$, attenuated antiviral responses ${ }^{34}$, leukocyte attrition, or mutation accumulation. Key genetic mutations with increased age include both somatic mutations and mosaic chromosomal alterations (mCAs), such as deletions, duplications, and loss of heterozygosity. A recent large study described how expanded mCA clones increased with age, were associated with altered leukocyte numbers, and showed significant associations with infections ${ }^{35}$. Intriguingly, this process may act synergistically with genetic variation, as GWASs have identified multiple variants in DNA-damage-repair pathways associated with $\mathrm{mCAs}{ }^{36}$. Likewise, postzygotic somatic mutations increase with age and can impact immune responses, with somatic variants frequently observed in the $\mathrm{CD} 8^{+} \mathrm{T}$ cells of people with multiple sclerosis or rheumatoid arthritis, and noncoding somatic variants that act as eQTLs have been described in cancer $^{37}$. The relative contributions of these factors largely remain to be dissected, and it is likely that many age-associated immune changes are driven by combinations of factors or through secondary changes that occur during aging, such as increased inflammation or pathogen exposure - for example, cytomegalovirus infection, which impacts multiple immune phenotypes ${ }^{12}$. It is also important to consider cumulative environmental exposures as potential confounders. Few detailed immune-phenotyping studies have been run outside of countries with historic economic privilege, and even within such countries, large-scale changes in environmental exposure separate older participants from younger participants.
Well-established age-associated changes may therefore reflect infectious and environmental history, rather than purely reporting on the direct effect of age itself.

\section{Sex and immune variation}

The effect of sex on immune variation is most apparent at the clinical level. There are differences between women and men in risks for immune-related diseases, including those linked to autoimmunity and viral infection. At the level of immune parameters, the most consistently observed associations with sex are altered baseline cellular traits and differential responses to vaccinations ${ }^{38}$. A recent study also found that $47 \%$ of HLA class I and II genes showed differences between sexes in expression following stimulation with lipolysaccharide (LPS), a far greater rate than the genome average ${ }^{39}$. While much of the effect of sex is unexplained, the best-studied drivers for this variation are sex chromosomes and sex hormones.

With an abundance of immune-related genes present on the $\mathrm{X}$ chromosome, the differential allosome allocation can explain many sex-associated immune differences. Several studies have shown biallelic expression of the X-encoded TLR7 in females, which results in higher levels of type I interferon induction by plasmacytoid dendritic cells and a greater propensity for immunoglobulin G (IgG) class switching in B cells ${ }^{40,41}$. By contrast, analyses of whole-blood transcriptional responses to LPS found that the vast majority of $\mathrm{X}$-linked genes are commonly induced in both males and females, including the majority of genes known to escape $\mathrm{X}$ inactivation ${ }^{39}$. TLR7 may thus represent a unique case, rather than a general rule. While the Y chromosome is genetically poor, there is evidence that mosaic loss of chromosome Y (LOY) contributes to immune variation. LOY is the most common postzygotic mCA in leukocytes and is associated with earlier mortality and morbidity in men. LOY is higher in innate cells than in adaptive cells and is associated with large-scale transcriptional dysregulation ${ }^{42}$. Whether these associations are direct and casual or reflect a common underlying mechanism requires further study.

Sex hormones are the other major source of potential sex-associated immune differences. Antibody responses to influenza vaccination positively correlate with plasma estradiol concentrations in females ${ }^{43}$ and negatively correlate with plasma testosterone in young males ${ }^{43,44}$. Studies of sex hormones are complicated by the intersection with age, as estradiol, progesterone, and testosterone levels fluctuate throughout life. It is therefore important to consider that immune variability may be specific to certain developmental periods in life, and that age effects may often be nonlinear, as was reported for differential immune response specifically to influenza H1N1 stimulation ${ }^{45}$ and vaccination ${ }^{46}$.

While substantial immune variation is associated with sex, almost no data are available to discriminate between the impact of sex and that of gender within these studies, and effects attributed to sex may, at least in part, be causally driven by gender. A large-scale blood transcriptomics analysis found sharply divergent effects of sex in an urban environment versus a rural environment in $\mathrm{Morocco}^{47}$. Immune differences between male and female participants were strongly amplified in the rural setting, with traditional gender roles altering environmental exposures, compared with those in the less gender-segregated urban setting ${ }^{47}$. This result implies that the primary effect observed was based on gender rather than sex, although the topic requires focused research. To definitively assign causation to sex-associated immune variation, future systems-immunology studies will need to be actively inclusive; engagement of immigrant and transgender populations might provide valuable insights into the sex versus gender effects. The strong correlation of sex with immunological clinical outcomes demonstrates the urgent clinical need of understanding the basis for this immune variation and improving the tailoring of medical strategies to these differences. 


\section{Environmental drivers of immune variation}

Adequate nutrition is essential for a functioning healthy immune response. Severe nutritional deficiencies lead to immune defects, in particular in children ${ }^{48}$. However, despite many claims, it remains unclear how normal dietary variation directly affects immune responses, or whether diet mediates its effects through indirect complex effects, such as changes in the microbiome (discussed below), body weight, or associated inflammatory effects. As an example, a recent systems-immunology study identified food-derived metabolites as potential drivers for an urban-rural divide in immune configurations $^{49}$, although environmental exposures might be confounders. Randomized placebo-controlled studies are therefore essential for assessing the direct effect of diet on immune function. A recent meta-analysis of 18 such studies of probiotic supplementation found only limited effects on immunity in healthy adults $s^{50}$, while a small-scale study identified fermented foods as reducing inflammatory markers ${ }^{51}$. Despite this, there is evidence for immunomodulatory roles of key dietary components. For example, iron deficiency is widespread in infants from lower-income countries and has been shown to be important for development of $\mathrm{T}$ cell and $\mathrm{B}$ cell responses. A retrospective study of iron deficiency in infants found that iron supplementation improved responses to measles vaccination $^{52}$. With supporting evidence from in vitro studies ${ }^{53}$ and people with mutations in TFRC, which encodes transferrin receptor 1 (ref. ${ }^{54}$ ), it is clear that iron bioavailability is necessary for efficient $\mathrm{B}$ cell responses. For other macronutrients, evidence exists for the importance of dietary salt, specifically for $\mathrm{T}_{\mathrm{H}} 17 \mathrm{immunity}$. High-salt diets have been associated with increased risk of autoimmunity ${ }^{55}$ and reduced mitochondrial function in monocytes ${ }^{56}$. In vitro and mouse experiments have identified increased $\mathrm{T}_{\mathrm{H}} 17$ cells as a potential mechanism linking high salt to autoimmunity ${ }^{57}$, supported by $\mathrm{T}_{\mathrm{H}} 17$ deficiencies in people with salt-losing tubulopathies ${ }^{58}$ and small-scale challenge experiments ${ }^{59}$. Finally, vitamin $\mathrm{D}$ is important for many physiological processes, and the use of placebo-controlled, randomized interventional studies is helping to identify which immune pathways are affected ${ }^{60}$. Despite these examples, and more, it remains unclear whether the relationships between nutrition and immunity are continuous, with population-level effects driven by different diets, or whether they exist only at the extreme ends of the spectrum, such as in iron deficiency or very-high-salt diets. Similarly, for body weight, while a moderate body-mass-index range has not been observed to be associated with multiple immune traits ${ }^{20}$, individuals with obesity have been reported to be deficient in natural killer cell numbers and function, driven by the lipid-rich environment ${ }^{61}$.

Environmental exposures are potential drivers of immune variation, with particulate matter in pollution and industrial chemicals found in food and our domestic and work environments capable of driving immune deviation ${ }^{62}$. Evidence for the immune-altering capacity of environmental exposure is strongest in the downstream clinical manifestations, where, for example, air pollution and industrial-chemical accidents are linked to inflammatory disease $^{63,64}$, and farm-animal exposure protects from asthma ${ }^{65}$. Combined with animal-exposure models and in vitro studies ${ }^{66}$, variation in exposure to pollutants and chemicals is likely to drive strong immune divergence. An example of the mechanistic link being directly made is the role of aryl hydrocarbons in promoting $\mathrm{T}_{\mathrm{H}} 17$ responses. Following the identification of the molecular basis ${ }^{67}$, positive correlations have been found between levels of particulate-matter air pollution and circulating $\mathrm{CCR}^{+} \mathrm{T}$ cells prone to $\mathrm{T}_{\mathrm{H}} 17$ polarization ${ }^{68}$. While the latter population association was performed in people with multiple sclerosis, it provides a clear proof of principle that variable environmental exposures can contribute to the immune configurations observed within a population. Challenge studies can also be used, such as replicating urban diesel-exhaust exposure in a healthy cohort, demonstrating
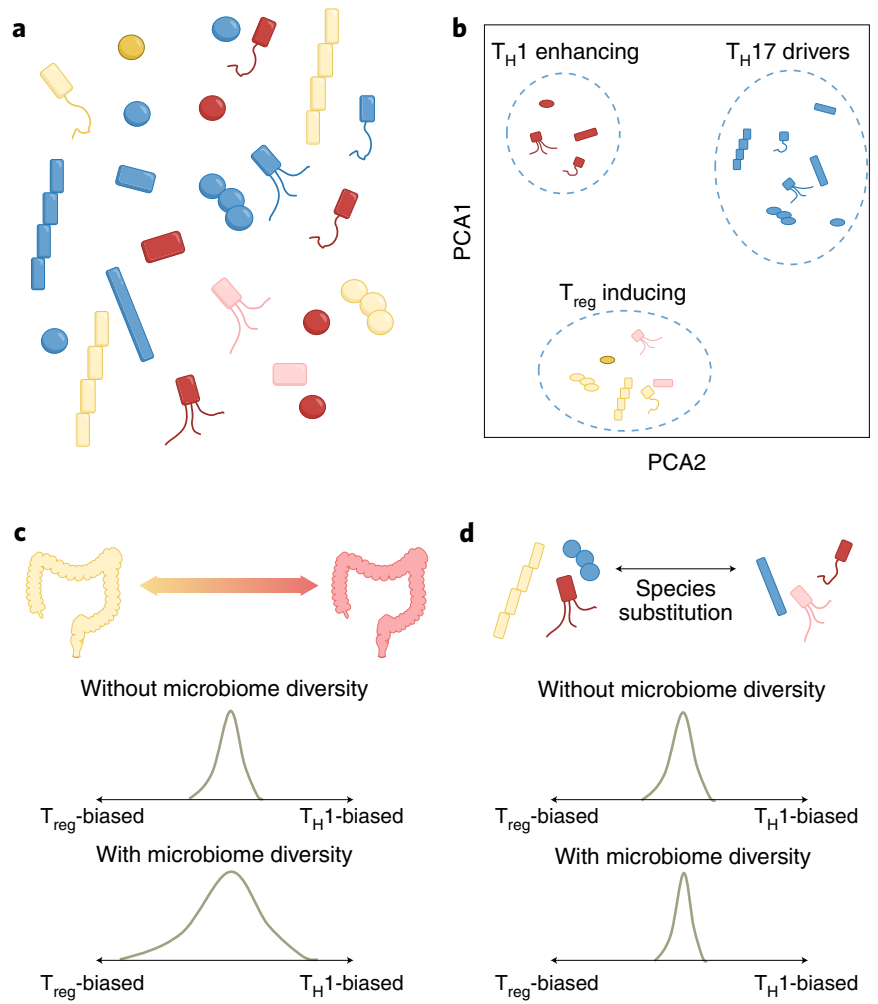

d
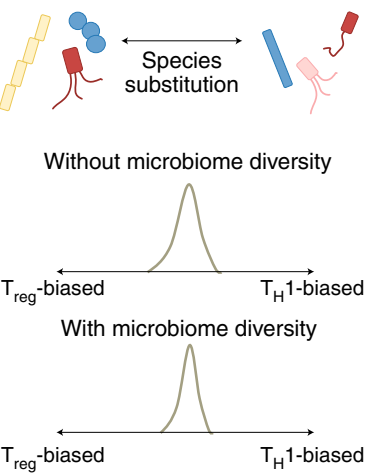

Fig. 2 | Simplified models for interaction of microbiome diversity and immune variation. $\mathbf{a}$, Within the human microbiome is a large diversity of commensal species, some of which will have the potential to drive immune phenotypes in different directions (illustrated with color). b, A hypothetical principal component analysis of immunome-active commensal species, based on their impact on the human immune system. Clusters illustrate different microbiome species that have similar immune-shaping properties. c, Under a model of colonization independence, individuals will vary in the relative representation of different commensals. When only a single axis of $T_{\text {reg }}$ cell-biasing and $T_{H} 1$ cell-biasing commensals is considered, individuals with a microbiome rich in an immune-shaping species set will have more extreme immune variation. The effect is a net expansion of immune variation across the human population. $\mathbf{d}$, Under a model of colonization interdependence, microbiome diversity is driven by species substitution. Loss of one immune-shaping commensal provides a niche that is filled by other commensals with similar immune-shaping potential. The net effect on immune variation across the human population is negligible under this model.

the resulting elevation of IgE-mediated responses ${ }^{69}$. Exposureelimination studies ${ }^{70}$ provide the reverse design. Among the most potent environmental exposures, due to its ubiquity, is cigarette smoking, which drives an inflammatory and possibly autoimmune state, with changes in many leukocyte cell types ${ }^{20}$. Understanding the effects of smoking is complicated by the over 4,000 toxic substances present. A better understanding of these complex effects may be provided through comparative studies with ex-smokers as well as individuals exposed to passive smoking, e-cigarettes, or nicotine products. While the effects of individual environmental exposures need to be explored, the net aggregate is potentially extremely potent, and may account for such findings as a halving of immune variation in cohabitating couples ${ }^{3}$.

The human microbiome has the potential to be a major contributor to immune variation. The microbiome exhibits an extraordinary degree of interindividual compositional diversity ${ }^{71}$. The presence of the microbiome influences immune development and function, with infant microbial colonization modifying immune 
development ${ }^{4,72}$, and adult broad-spectrum antibiotic treatment truncating vaccine responses ${ }^{73}$. Among the strongest evidence for microbiome variation influencing immune states is the association with diseases. Shifts in microbiome composition are observed in different inflammatory and immunological diseases. While such associations may be correlative in nature, microbiome 'transplants' have demonstrated partial success, consistent with a causative relationship. Fecal microbiota transplantation following Clostridium difficile infection, the most promising condition for microbiome transplantation ${ }^{74}$, is accompanied by reduced inflammatory parameters $^{75}$. Isolated gut-microbiome species have been shown to promote immune biases toward $\mathrm{T}_{\mathrm{H}} 1 \mathrm{cell}^{76}, \mathrm{~T}_{\mathrm{H}} 17$ cell $^{77}$, or regulatory $\mathrm{T}$ cell $\left(\mathrm{T}_{\text {reg }}\right.$ cell $)$ differentiation ${ }^{78}$ when transferred into mice with limited or no resident microflora. Parallel shifts in microbiome and immune profiles have been observed in infants $s^{4,72}$ and the posthematopoietic stem-cell transplantation setting ${ }^{79}$, and microbiome supplementation in these settings alters the immune system ${ }^{72,80,81}$. Together, these data strongly suggest that, at least in individuals with dysbiosis or limited microbiome diversity, introduction of new species does modify the immune status.

Of the diverse mechanisms by which variation in the microbiome may influence host immune variation, several have been experimentally validated. First, commensals provide antigenic targets $^{82}$. While the impact of such responses will be diluted above the clonal level, the immune response at the interface tissue is likely to be profoundly shaped by these interactions, as has recently been demonstrated in the clonal dominance in tissues observed after vaccine challenge ${ }^{83}$. Second, the microbiome produces bioactive metabolic products, which can shape the host immune system. For example, short-chain fatty acids are implicated in the ability to induce $\mathrm{T}_{\text {reg }}$ cells in mice ${ }^{78}$, and tryptophan metabolites reduce interferon- $\gamma$ responses by human cells in vitro ${ }^{84}$. The key unknown is the extent to which these immunomodulatory properties are amplified or negated across the microbiome variation observed in the healthy human population. If independence in microbial colonization efficiency occurs, these effects would drive a spectrum of immune-modifying microbiomes, increasing variance in immune configurations. Conversely, interdependency in microbial colonization may result in niche substitution, limiting the overall impact of microbiome diversity on immune variation (Fig. 2). Notably, the initial immune variation among infants converges as the microbiome stabilizes, independently of gestational age ${ }^{85}$, suggesting there is some degree of interdependency in the effects of colonization ${ }^{4}$. Large-scale studies with parallel systems immunology and microbiomics, such as one identifying that up to $10 \%$ of the variation in cytokine responses could be accounted for by the microbiome ${ }^{84}$, are needed to answer such questions.

The balance of probabilities suggests that microbiome variation between individuals contributes to the observed immune diversity. The magnitude of this contribution, however, remains to be determined. The responsiveness of the microbiome to diet, environmental exposure, age, and $\operatorname{sex}^{86,87}$ creates problematic confounders. Even in the case of GWASs, in which associations found with the microbiome show some overlap with immunological loci, reproducibility has been problematic, and the direction of causality between immune traits and microbiome traits associated with the same loci has not been established ${ }^{88}$. In each case, it remains possible that microbiome changes are largely bystander correlations, responding in parallel with the immune system to the causative drivers. Equally, the microbiome may be the nexus that integrates many of the associated variables and provides the direct causative mechanisms underlying immunological variation.

\section{Variation during immune reactions}

As an emerging field of research, systems immunology has concentrated largely on understanding the nature of variation in the healthy baseline state. Beneath this baseline variation, however, lies a hidden layer of immune variation, present only during immune responses. Indeed, it is precisely the outcome of immune challenge that has shaped the evolution of our immune system. Systems-vaccinology studies are the key approach to investigating the perturbation of the immune system in a controlled manner, with influenza vaccination being among the most extensively studied. Variation in the production of protective antibodies following vaccination has been correlated with high plasmablast activity within a week after vaccination ${ }^{89,90}$. The presence of $\mathrm{CD} 38^{+} \mathrm{B}$ cell subsets at baseline seems to be a strong predictor across diverse cohorts and studies ${ }^{89,91}$, with specific gene-expression signatures ${ }^{89,91-93}$. Variation in the early activation of the interferon pathway has been reproducibly associated with elevated antibody production in the latter response ${ }^{89-91,94}$, a finding consistent across multiple different vaccines ${ }^{91,94-96}$. These positive innate responses can also be found at baseline and are mediated mainly by dendritic cells and plasmacytoid dendritic cells $^{91,97}$. Intriguingly, a similar interferon signature correlates with prediction of clinical flares of systemic lupus erythematosus ${ }^{91}$, suggesting a common basis for an immunological variation that may have beneficial or detrimental consequences depending on the activation context.

A strong case study for the utility of systems-vaccinology approaches is the application of these lessons to understanding immune differences with age. The functional correlation between early interferon responses and antibody production in healthy young individuals may suggest the causality of poor immune response after vaccination in the aged population ${ }^{90,92}$. The aged population, in comparison with younger individuals, has an innate cell compartment skewed toward an inflammatory signature but away from type I interferons ${ }^{90,93,98,99}$. Indeed, the addition of an interferon-stimulating adjuvant to an influenza vaccine substantially improves the production of antibodies in older people ${ }^{100,101}$. Studying natural variation between good responders and poor responders therefore identifies target pathways that can be exploited for improving clinical outcomes in the poor responder population.

Although vaccines constitute the best-controlled systems-immunology challenge context, experimental and natural infections provide the most physiologically relevant. The SARS-CoV-2 pandemic has provided the most intensively studied natural infection context, with a multitude of systems-immunology studies identifying immunological variations associated with protection from severe infection. Clonal cross-reactivity explains a proportion of protection ${ }^{102,103}$; however, other factors have been linked to differential immunological response configurations. Cytokine bias, driven by an altered myeloid compartment ${ }^{104-106}$, appears to significantly alter the risk of severe disease, with type I interferons being protective and IL- 6 or TNF being detrimental ${ }^{104,105,107}$. In the lymphoid compartment, susceptibility to severe disease is associated with elevated activation and clonal expansion of $\mathrm{CD}^{+} \mathrm{T}$ cells ${ }^{104,108,109}$. The contribution of this variation in immune responses to clinical outcomes is emphasized by the predictive nature of early immune configurations for later pathology ${ }^{109,110}$. While some component of the association between immune status and clinical outcome may be shared across infections, especially those sharing infectious modalities $^{111}$, other associations are likely to differ. An example of the latter is the association of $\mathrm{CD} 4^{+} \mathrm{T}$ cell responses with parasitemia restraint in controlled malaria infections ${ }^{112}$. The utility of systems immunology in understanding clinical outcomes in SARS-CoV-2 infections is likely to drive the uptake of this approach to other infectious diseases. We anticipate that different immune configurations will each provide either beneficial effects or detrimental effects, depending on the infectious challenge.

Regulatory QTL mapping during dynamic processes such as the response to immune stimuli can reveal otherwise hidden regulatory variation that may be particularly relevant for disease. As 


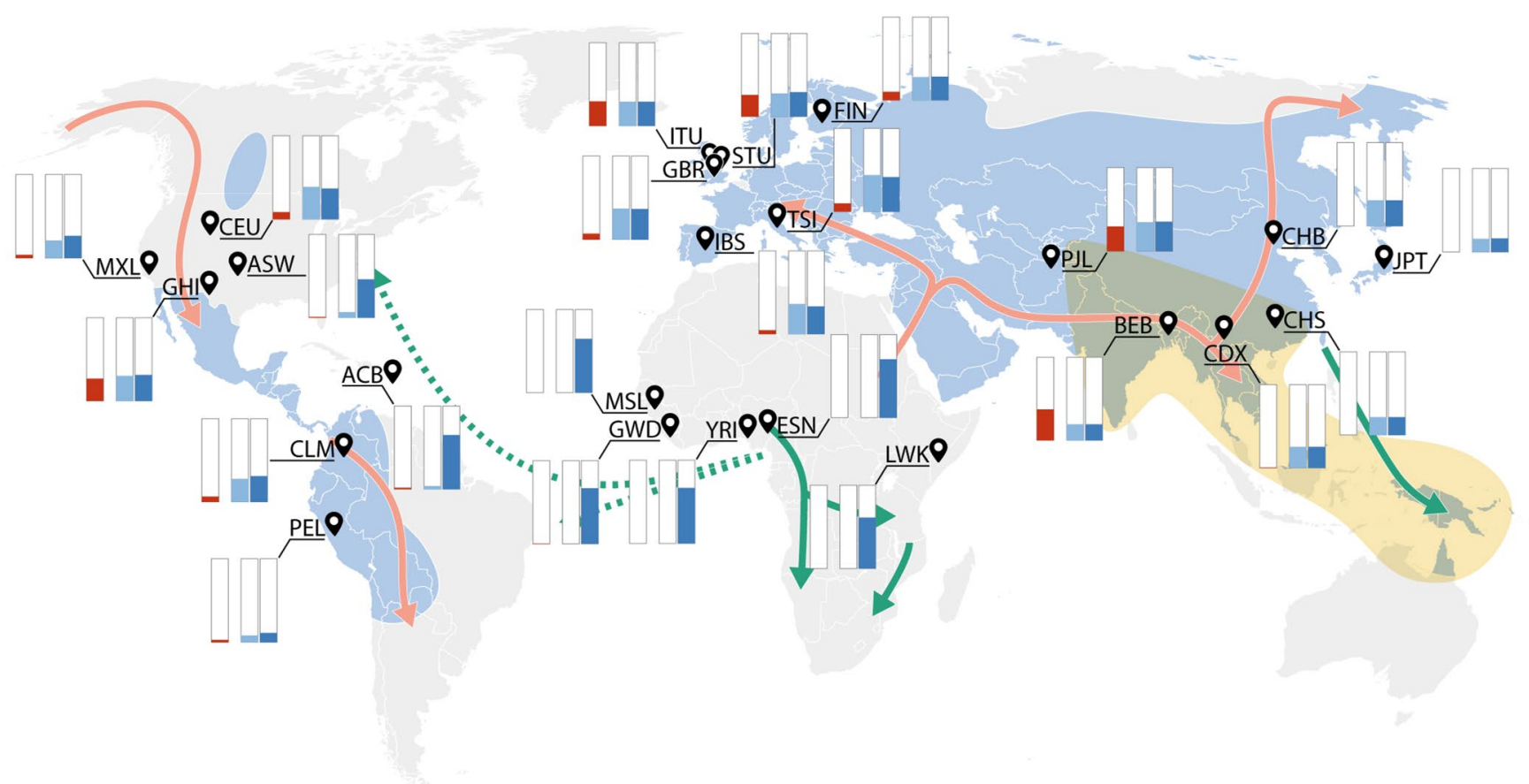

Fig. 3 | Neanderthal ancestry impacts COVID-19 severity. The major risk factor for severe COVID-19, which almost doubles the risk, is a Neanderthal haplotype block on chromosome 3 encompassing the gens encoding the chemokine receptors CXCR6 and CCR9 (tagged by rs35044562, red), which is especially common in South Asians. A different Neanderthal haplotype block, at the OAS1-OAS3 cluster on chromosome 12 (tagged by rs1156361, light blue), is protective against severe COVID-19 in Eurasians. This haplotype appears to have reintroduced the ancestral OAS1 splice variant rs10774671 (dark blue), still present as an isolated variant in Africans. Frequencies indicated by bars for 1000 Genomes Project populations: ACB, African Caribbean; ASW, African ancestry in the southwest United States; BEB, Bengali (Bangladesh); CDX, Dai Chinese; CEU, Northern and Western European ancestry in the United States; CHB, Han Chinese; CHS, Southern Han Chinese; CLM, Colombian; ESN, Esan (Nigeria); FIN, Finnish; GBR, British; GIH, Gujarati Indians in Houston (United States); GWD, Gambian Mandika; IBS, Iberian (Spain); ITU, Indian Telugu in the United Kingdom; JPT, Japanese; KHV, Kinh Vietnamese; LWK, Luhya (Kenia); MSL, Mende (Sierra Leone); MXL, Mexican ancestry in California (United States); PEL, Peruvian; PJL, Punjabi (Pakistan); PUR, Puerto Rican; STU, Sri Lankan Tamil (United Kingdom); TSI, Toscani (Italy); YRI, Yoruba (Nigeria). Adapted population genomics map ${ }^{134}$ : orange arrows indicate the major migrations of Homo sapiens after the out-of-Africa exodus; green arrows indicate some more recent migratory events. Approximate geographic areas of modern human populations presenting Neanderthal or Denisovan ancestry are shaded in light blue and yellow, with the Neanderthal ancestry observed in American populations reflecting their varying levels of European ancestry.

reviewed elsewhere ${ }^{24}$, immune cell activation reveals a large number of trans-acting response eQTLs, which are highly cell type specific. The response eQTLs identified in the context of pathogen sensing can have large effect sizes, and show a stronger enrichment for immunological disease associations and evidence of recent selection than do standard, steady-state eQTLs ${ }^{24,45,113}$. Transcriptional variation is to a large extent buffered at the protein level, and the first GWASs for pQTLs after in vitro stimulation are emerging ${ }^{14,114,115}$. Significantly increased interindividual variability in cytokine response has been observed following stimulation ${ }^{115}$, and is under strong genetic control and typically pathogen centered rather than cytokine centered ${ }^{14,114,115}$. As in the previously mentioned baseline example, nearly all pQTLs are trans effects ${ }^{14,114,115}$, and they are enriched for infectious-disease associations ${ }^{14,114,115}$. Human genetic determinants following in vivo encounters have also been studied. Several GWASs have been performed for response to vaccination, although further replication is needed before firm conclusions can be drawn, and studies are increasingly being performed on responses to infections ${ }^{116}$. While associations with clinical outcome have been reviewed elsewhere, the response to SARS-CoV-2 is worth noting, as the COVID-19 pandemic provided a recent opportunity for infectious-outcome GWASs that are orders of magnitude larger than previous studies. These studies have identified 13 genome-wide significant loci with relatively large effect sizes associated with infection or clinical outcome ${ }^{117-119}$. Very few studies, however, have included immunological phenotypes during an in vivo infection-response GWAS. One well-known early example was the association of interferon- $\lambda 4$ expression with clearance of hepatitis $C$ virus. In a genome-to-genome analysis, this host variation was subsequently demonstrated to drive viral polymorphism and viral load ${ }^{120}$. Here, again, the translational potential exists to use the correlation between immune variation and infectious outcome as a rational guide to therapeutic intervention.

\section{The continuous evolution of immune variation}

The evolutionary advantages of immune variation are imprinted upon our genomes. From human origins to modernity, the signature of selection for immunological genetic variants is evident. Among the most fascinating historic selection events is the archaic introgression of variants from Neanderthal and Denisovan genomes into those of modern humans. Neanderthal ancestry accounts for $\sim 2 \%$ of the genome of Euroasians, while Denisovan ancestry represents $<1 \%$ of the genome in East and South East Asians and up to $6 \%$ in some Oceanian populations. Despite the widespread signature of purifying selection against archaic alleles, selection acting on advantageous archaic introgressed segments may have increased their frequency ${ }^{8}$. Introgressed Neanderthal and Denisovian loci are enriched for innate and adaptive immunity genes ${ }^{121,122}$, and include the HLA and immunoglobulin regions ${ }^{123,124}$. RNA viruses appear to have been an important driving force of such positive selection, with up to $30 \%$ of high-frequency Neanderthal introgressions being selected in response to viruses ${ }^{125}$. Between $46 \%$ and $65 \%$ of alleles 
a

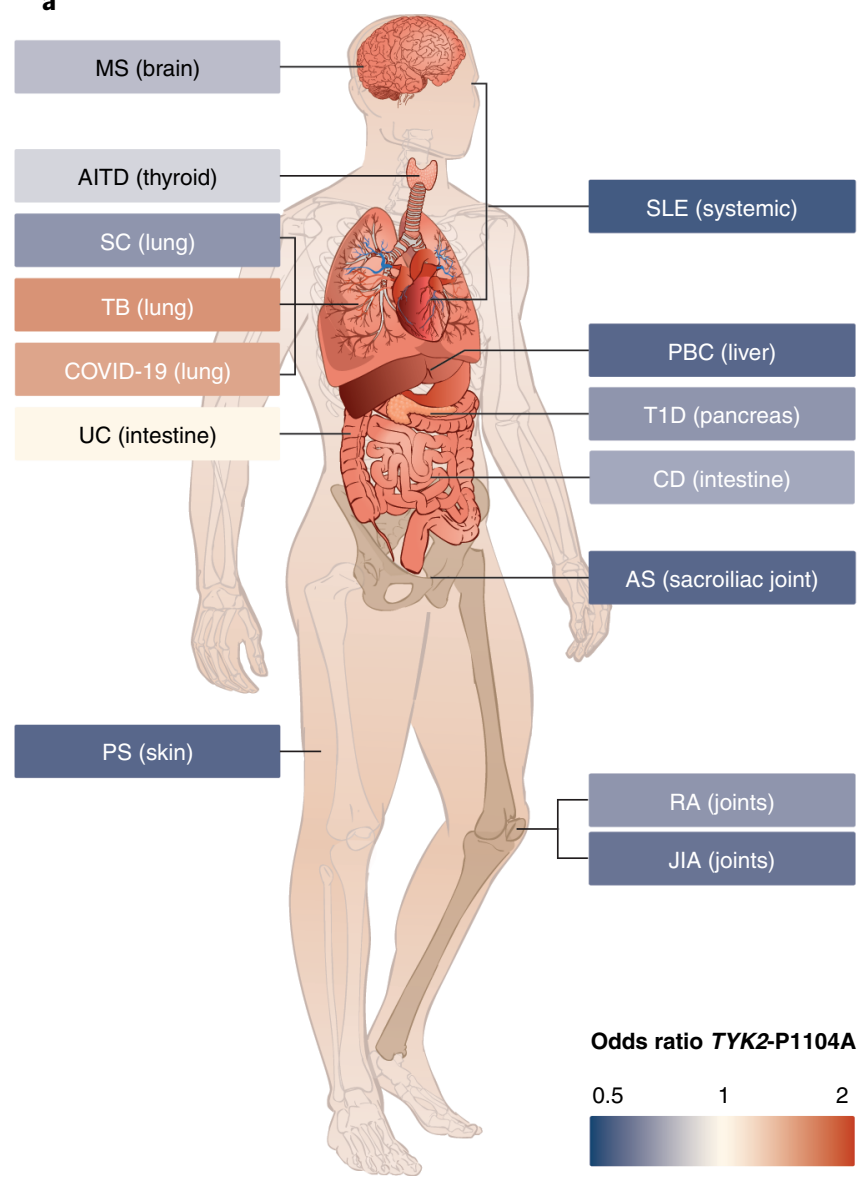

b

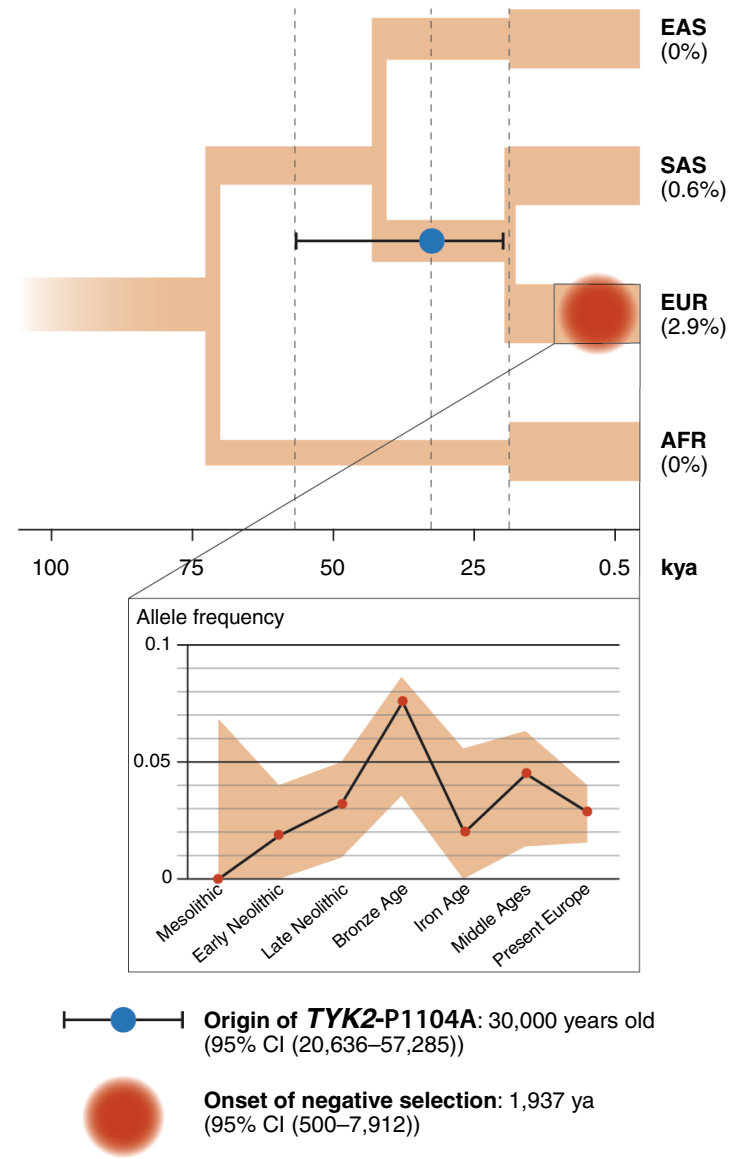

Fig. 4 | Immune variation exerts context-dependent opposite effects on disease. a, TYK2-P1104A (rs34536443) is a unique shared protective variant for more than ten autoimmune diseases, whereas it is the main common risk factor for tuberculosis in endemic regions and a variant in high linkage disequilibrium increases the risk of severe COVID-19. The degree of protection or risk is based on the published allele-dosage model ${ }^{130,131}$, GWAS EBI catalog (June 2021) and GenOMICC Consortium (release 6, 15 June 2021), with a recessive model exerting even larger effects for those diseases for which it has been tested. AITD, autoimmune thyroid disease; AS, ankylosing spondylitis; CD, Crohn's disease; JIA, juvenile idiopathic arthritis; MS, multiple sclerosis; PBC, primary biliary cirrhosis; PS, psoriasis; RA, rheumatoid arthritis; SC, sarcoidosis; SLE, systemic lupus erythematosus; T1D, type 1 diabetes; TB, tuberculosis; UC, ulcerative colitis. b. The TYK2-P1104A variant originated around 30,000 years ago, with present frequencies in the indicated major 1000 Genomes populations. The variant has undergone strong negative selection in Europeans over the past 2,000 years, and this has been suggested to have co-occurred with the emergence of Mycobacterium tuberculosis as one of the most deadly diseases in Europe. Figure adapted from published results ${ }^{132}$, listing populations and frequencies from the 1000 Genomes Project. AFR, sub-Saharan African; EUR, European; SAS, South Asian; EAS, East Asian; kya, thousand years ago.

in introgressed haplotypes represent ancient genetic diversity lost in the out-of-Africa bottleneck, and for $70 \%$ of these alleles reintroduced in Eurasians, the ancestral form is still present in Africans ${ }^{126}$, demonstrating the selective pressure for maintaining genetic diversity in immunity.

Increasing evidence for functional consequences to introgressed alleles is being found. The example of FCGR2A (which encodes an immunoglobulin receptor) underscores the importance of alternative splicing also in response to infection. An introgressed variant, in linkage disequilibrium with immune-trait-associated signals $^{20}$, increases protein-coding transcripts and phagocytosis after stimulation by LPS $^{26}$. The role of introgression in shaping the genetic architecture of COVID-19 is notable. The major risk factor for severe COVID-19, almost doubling the risk, is a haplotype block encompassing the genes encoding the chemokine receptors CXCR6 and CCR9 (refs. ${ }^{117-119}$ ). This haplotype has been introduced by archaic introgression ${ }^{124}$ (Fig. 3). The high frequency of this Neanderthal haplotype in Europeans (8\%) and South Asians $(30 \%)$ suggests that the variant previously underwent positive selection $^{124,127}$, raising the possibility of oscillating selection with changing infectious exposures over time. Alternatively, the striking difference in frequency between South Asia and East Asia, where the variant is largely absent, has been suggested to result from negative selection, perhaps from coronaviruses or other pathogens ${ }^{127}$. A different Neanderthal haplotype block, at the OAS1-OAS3 cluster, is protective against severe COVID-19 in Eurasians ${ }^{117-119}$, producing an OAS1 splice form with higher enzymatic activity ${ }^{128}$. The ancestral variant, still present in Africans, appears to confer a similar magnitude of protection against severe COVID-19, underscoring the value of the reintroduction ${ }^{129}$.

The evolution of immunity has not halted with modernity. While historical immune evolutionary processes were driven primarily by the burden of infectious disease, and in particular childhood mortality from infections, the shifting context of immune challenges still provides selective pressure. The plethora of immune-driven pathologies, each at risk of amplification by particular immune configurations, creates complex environment-dependent trade-offs. Genetic determinants of immune variation overlap known immune disease 


\section{Box 1 | Future challenges}

- Development of standardized technical and analytical systems immunology platforms to allow meta-analyses of studies

- Inclusion of diverse under-represented ethnicities into GWAS

- Disentangling the overlap of genetic association signals between immunological traits and clinical outcomes

- Identifying the cumulative magnitude of rare variants on immunological diversity at a population level

- Application of genetic approaches to dissect the mechanistic basis of immune trait associations

- Extending systems-immunology and systems-vaccinology studies beyond the context of historically wealthy countries to allow cross-comparison of healthy aging

- Engagement with the trans community and other populations that will allow dissection of the effects of sex and gender

- Large-scale studies combining systems immunology, microbiome assessment, and environmental-exposure analysis to unravel questions of causality

- Increased experimental medicine studies (such as vaccination, antibiotic treatment, interventional cross over clinical study designs) to test causality of associations

loci ${ }^{9-11,18,19,29,115}$. As examples, association signals for immunoglobulin levels overlap genes known to be involved in autoimmune and immunodeficiencies ${ }^{29}$, monocyte-derived cytokine associations overlap infectious diseases, and $\mathrm{T}$ cell-derived cytokine associations overlap autoimmune disease $\mathrm{s}^{14,115}$. The nature of immune variation suggests that no variants will be unambiguously beneficial, with instead each immune configuration bias incurring both a benefit and a penalty, in a context-dependent manner. The arrival of an era of lower infectious-disease burdens, in particular the hygienic control of fecal-oral transmission, childhood vaccination, and antibodies, has not eliminated the selective pressure on the immune system, but has instead changed the relative benefits and penalty of particular variants. An example of this principle may be seen in the P1104A variant of TYK2, a uniquely broad immune-disease locus. Homozygosity for this variant confers susceptibility for severe mycobacterial disease, most frequently tuberculosis ${ }^{130}$. At the same time, P1104A homozygosity is associated with fivefold protection against multiple autoimmune diseases ${ }^{131}$ and changes in leukocyte frequency ${ }^{16,17}$ (Fig. 4a). The allele frequency declined from $\sim 9 \%$ in prehistoric humans to $\sim 3-4 \%$ in Europeans, among the highest purifying selection observed in the human genome $e^{132}$ (Fig. 4b). The recent decline in tuberculosis prevalence has eliminated this association in Europe ${ }^{131,133}$ and has prevented further negative selection of the allele. However, the recent emergence of SARS-CoV-2 may expose the adverse functional consequences of TYK2 genetic variation once more. An intergenic variant in strong linkage disequilibrium with P1104 is associated with COVID-19 severity ${ }^{117,119}$. The effect of this allele therefore balances increased risk of certain infections with protection from autoimmunity in a temporally and spatially dependent manner.

\section{Conclusion}

The genetic architecture of immune diversity and capacity for malleability lies in our evolutionary history. While the rate of natural-selection-induced genetic change is reduced, the changing environment is altering the physiological consequences of this archaic genetic variation. Systems-immunology and systems-vaccinology approaches have made great advances in recent years in elucidating the basic structure of this variation.
Multiple key challenges still remain in dissecting causal mechanisms (see Box 1), with engagement of neglected populations and the joint application of techniques from genomics, population genetics, microbiomics, and environmental epidemiology being critical for further progress. Beyond the mechanistic understanding of natural variation lies the promise of identifying individuals with potentially pathogenic immune configurations and the use of therapeutics to reroute immunity into a healthy state. Natural variation in the immune system highlights the pathways that are amenable to large functional shifts after modulation. Understanding variation in response to environmental factors in particular, such as diet, microbiome, and environmental exposure, holds the promise of using simple environmental manipulations in a targeted manner to reroute an individual's immune system toward a less pathogenic configuration. Although the advantages of personalized immune modification are manifold, they first require a baseline knowledge of the source of our individual differences.

Received: 28 July 2021; Accepted: 17 September 2021; Published online: 18 November 2021

\section{References}

1. Kaczorowski, K. J. et al. Continuous immunotypes describe human immune variation and predict diverse responses. Proc. Natl Acad. Sci. USA 114, E6097-E6106 (2017).

2. Lakshmikanth, T. et al. Human immune system variation during 1 year. Cell Rep. 32, 107923 (2020).

3. Carr, E. J. et al. The cellular composition of the human immune system is shaped by age and cohabitation. Nat. Immunol. 17, 461-468 (2016).

4. Olin, A. et al. Stereotypic immune system development in newborn children. Cell 174, 1277-1292 (2018).

5. Cheung, P. et al. Single-cell chromatin modification profiling reveals increased epigenetic variations with aging. Cell 173, 1385-1397 (2018).

6. Colbran, L. L. et al. Inferred divergent gene regulation in archaic hominins reveals potential phenotypic differences. Nat. Ecol. Evol. 3, 1598-1606 (2019). Genetic information is able to predict phenotypic differences between archaic hominins and between archaic hominins and anatomically modern humans.

7. Sullivan, R. J. \& Weber, J. S. Immune-related toxicities of checkpoint inhibitors: mechanisms and mitigation strategies. Nat. Rev. Drug Discov. https://doi.org/10.1038/s41573-021-00259-5 (2021).

8. Quintana-Murci, L. Human immunology through the lens of evolutionary genetics. Cell 177, 184-199 (2019).

9. Orru, V. et al. Genetic variants regulating immune cell levels in health and disease. Cell 155, 242-256 (2013). A multigenerational study demonstrating the heritability of cellular immune traits and was the first GWAS identifying genetic drivers.

10. Orru, V. et al. Complex genetic signatures in immune cells underlie autoimmunity and inform therapy. Nat. Genet. 52, 1036-1045 (2020).

11. Roederer, M. et al. The genetic architecture of the human immune system: a bioresource for autoimmunity and disease pathogenesis. Cell 161, 387-403 (2015).

12. Brodin, P. et al. Variation in the human immune system is largely driven by non-heritable influences. Cell 160, 37-47 (2015).

13. Mangino, M., Roederer, M., Beddall, M. H., Nestle, F. O. \& Spector, T. D. Innate and adaptive immune traits are differentially affected by genetic and environmental factors. Nat. Commun. 8, 13850 (2017).

14. Li, Y. et al. A functional genomics approach to understand variation in cytokine production in humans. Cell 167, 1099-1110 (2016).

15. Liu, Y. et al. Quantitative variability of 342 plasma proteins in a human twin population. Mol. Syst. Biol. 11, 786 (2015).

16. Vuckovic, D. et al. The polygenic and monogenic basis of blood traits and diseases. Cell 182, 1214-1231 (2020).

17. Chen, M. H. et al. Trans-ethnic and ancestry-specific blood-cell genetics in 746,667 individuals from 5 global populations. Cell 182, 1198-1213 (2020). This very large GWAS identifies thousands of genetic variants underlying basic hematological traits and illustrates the power of trans-ethnic analyses.

18. Aguirre-Gamboa, R. et al. Differential effects of environmental and genetic factors on T and B cell immune traits. Cell Rep. 17, 2474-2487 (2016).

19. Lagou, V. et al. Genetic architecture of adaptive immune system identifies key immune regulators. Cell Rep. 25, 798-810 e796 (2018).

20. Patin, E. et al. Natural variation in the parameters of innate immune cells is preferentially driven by genetic factors. Nat. Immunol. 19, 302-314 (2018).

21. Li, S. et al. Molecular signatures of antibody responses derived from a systems biology study of five human vaccines. Nat. Immunol. 15, 195-204 (2014). 
22. Ahola-Olli, A. V. et al. Genome-wide association study identifies 27 loci influencing concentrations of circulating cytokines and growth factors. Am. J. Hum. Genet. 100, 40-50 (2017).

23. Sliz, E. et al. Genome-wide association study identifies seven novel loci associating with circulating cytokines and cell adhesion molecules in Finns. J. Med. Genet. 56, 607-616 (2019).

24. Umans, B. D., Battle, A. \& Gilad, Y. Where are the disease-associated eQTLs? Trends Genet. 37, 109-124 (2021).

25. Ota, M. et al. Dynamic landscape of immune cell-specific gene regulation in immune-mediated diseases. Cell 184, 3006-3021 (2021). The gene-regulation atlas of 28 immune cell types shows the dynamics of gene regulation depending on cell types and immunological conditions.

26. Rotival, M., Quach, H. \& Quintana-Murci, L. Defining the genetic and evolutionary architecture of alternative splicing in response to infection. Nat. Commun. 10, 1671 (2019). Differential isoform usage has been an important substrate of diversity in human immune responses and has been shaped throughout evolution and modernity.

27. Meyer, D., VR, C. A., Bitarello, B. D., DY, C. B. \& Nunes, K. A genomic perspective on HLA evolution. Immunogenetics 70, 5-27 (2018).

28. Dendrou, C. A., Petersen, J., Rossjohn, J. \& Fugger, L. HLA variation and disease. Nat. Rev. Immunol. 18, 325-339 (2018).

29. Jonsson, S. et al. Identification of sequence variants influencing immunoglobulin levels. Nat. Genet. 49, 1182-1191 (2017).

30. Pang, W. W. et al. Human bone marrow hematopoietic stem cells are increased in frequency and myeloid-biased with age. Proc. Natl Acad. Sci. USA 108, 20012-20017 (2011).

31. He, H. et al. Aging-induced IL27Ra signaling impairs hematopoietic stem cells. Blood 136, 183-198 (2020).

32. Webb, L. M. C. et al. Ageing promotes early T follicular helper cell differentiation by modulating expression of RBPJ. Aging Cell $\mathbf{2 0}$ e13295 (2021)

33. Clave, E. et al. Human thymopoiesis is influenced by a common genetic variant within the TCRA-TCRD locus. Sci. Transl. Med. 10, eaao2966 (2018).

34. Kohli, J., Veenstra, I. \& Demaria, M. The struggle of a good friend getting old: cellular senescence in viral responses and therapy. EMBO Rep. 22, e52243 (2021).

35. Zekavat, S. M. et al. Hematopoietic mosaic chromosomal alterations increase the risk for diverse types of infection. Nat. Med. 27, 1012-1024 (2021).

36. Thompson, D. J. et al. Genetic predisposition to mosaic Y chromosome loss in blood. Nature 575, 652-657 (2019).

37. Van Horebeek, L., Dubois, B. \& Goris, A. Somatic variants: new kids on the block in human immunogenetics. Trends Genet. 35, 935-947 (2019).

38. Flanagan, K. L., Fink, A. L., Plebanski, M. \& Klein, S. L. Sex and gender differences in the outcomes of vaccination over the life course. Annu. Rev. Cell Dev. Biol. 33, 577-599 (2017).

39. Stein, M. M. et al. Sex-specific differences in peripheral blood leukocyte transcriptional response to LPS are enriched for HLA region and X chromosome genes. Sci. Rep. 11, 1107 (2021).

40. Souyris, M. et al. TLR7 escapes X chromosome inactivation in immune cells. Sci. Immunol. 3, eaap8855 (2018).

41. Hagen, S. H. et al. Heterogeneous escape from $\mathrm{X}$ chromosome inactivation results in sex differences in type I IFN responses at the single human $\mathrm{pDC}$ level. Cell Rep. 33, 108485 (2020).

42. Dumanski, J. P. et al. Immune cells lacking Y chromosome show dysregulation of autosomal gene expression. Cell. Mol. Life Sci. 78 4019-4033 (2021)

43. Potluri, T. et al. Age-associated changes in the impact of sex steroids on influenza vaccine responses in males and females. NPJ Vaccines 4 29 (2019).

44. Furman, D. et al. Systems analysis of sex differences reveals an immunosuppressive role for testosterone in the response to influenza vaccination. Proc. Natl Acad. Sci. USA 111, 869-874 (2014).

45. Piasecka, B. et al. Distinctive roles of age, sex, and genetics in shaping transcriptional variation of human immune responses to microbial challenges. Proc. Natl Acad. Sci. USA 115, E488-E497 (2018).

46. Sobolev, O. et al. Adjuvanted influenza-H1N1 vaccination reveals lymphoid signatures of age-dependent early responses and of clinical adverse events. Nat. Immunol. 17, 204-213 (2016).

47. Idaghdour, Y. et al. Geographical genomics of human leukocyte gene expression variation in southern Morocco. Nat. Genet. 42, 62-67 (2010)

48. Cunningham-Rundles, S., McNeeley, D. F. \& Moon, A. Mechanisms of nutrient modulation of the immune response. J. Allergy Clin. Immunol. 115, 1119-1128 (2005). quiz 1129.

49. Temba, G. S. et al. Urban living in healthy Tanzanians is associated with an inflammatory status driven by dietary and metabolic changes. Nat. Immunol. 22, 287-300 (2021). A large-scale systems immunology study identified immune signatures associated with urban versus rural status in
Tanzania. Linear modelling suggested key changes were driven by food-derived metabolites.

50. Mohr, A. E., Basile, A. J., Crawford, M. S., Sweazea, K. L. \& Carpenter, K. C. Probiotic supplementation has a limited effect on circulating immune and inflammatory markers in healthy adults: a systematic review of randomized controlled trials. J. Acad. Nutr. Diet. 120, 548-564 (2020).

51. Wastyk, H. C. et al. Gut-microbiota-targeted diets modulate human immune status. Cell 184, 4137-4153 (2021).

52. Stoffel, N. U. et al. Iron deficiency anemia at time of vaccination predicts decreased vaccine response and iron supplementation at time of vaccination increases humoral vaccine response: a birth cohort study and a randomized trial follow-up study in Kenyan infants. Front Immunol. 11, 1313 (2020).

53. Hill, D. L. et al. Immune system development varies according to age, location, and anemia in African children. Sci. Transl. Med. 12, eaaw9522 (2020).

54. Jabara, H. H. et al. A missense mutation in TFRC, encoding transferrin receptor 1, causes combined immunodeficiency. Nat. Genet. 48, 74-78 (2016).

55. Toussirot, E., Bereau, M., Vauchy, C. \& Saas, P. Could sodium chloride be an environmental trigger for immune-mediated diseases? An overview of the experimental and clinical evidence. Front Physiol. 9, 440 (2018).

56. Geisberger, S. et al. Salt transiently inhibits mitochondrial energetics in mononuclear phagocytes. Circulation 144, 144-158 (2021).

57. Kleinewietfeld, M. et al. Sodium chloride drives autoimmune disease by the induction of pathogenic $\mathrm{T}_{\mathrm{H}} 17$ cells. Nature 496, 518-522 (2013).

58. Evans, R. D. R. et al. Inherited salt-losing tubulopathies are associated with immunodeficiency due to impaired IL-17 responses. Nat. Commun. 11, 4368 (2020).

59. Wilck, N. et al. Salt-responsive gut commensal modulates $\mathrm{T}_{\mathrm{H}} 17$ axis and disease. Nature 551, 585-589 (2017).

60. Goncalves-Mendes, N. et al. Impact of vitamin D supplementation on influenza vaccine response and immune functions in deficient elderly persons: a randomized placebo-controlled trial. Front. Immunol. 10, 65 (2019).

61. Michelet, X. et al. Metabolic reprogramming of natural killer cells in obesity limits antitumor responses. Nat. Immunol. 19, 1330-1340 (2018).

62. Vojdani, A., Ghoneum, M. \& Brautbar, N. Immune alteration associated with exposure to toxic chemicals. Toxicol. Ind. Health 8, 239-254 (1992).

63. van Voorhis, M. et al. Exposure to atmospheric particulate matter enhances $\mathrm{T}_{\mathrm{H}} 17$ polarization through the aryl hydrocarbon receptor. PLoS ONE 8, e82545 (2013)

64. Consonni, D. et al. Mortality in a population exposed to dioxin after the Seveso, Italy, accident in 1976: 25 years of follow-up. Am. J. Epidemiol. 167, 847-858 (2008).

65. Stein, M. M. et al. Innate immunity and asthma risk in Amish and Hutterite farm children. N. Engl. J. Med. 375, 411-421 (2016).

66. Suzuki, T., Hidaka, T., Kumagai, Y. \& Yamamoto, M. Environmental pollutants and the immune response. Nat. Immunol. 21, 1486-1495 (2020).

67. Veldhoen, $M$. et al. The aryl hydrocarbon receptor links $\mathrm{T}_{\mathrm{H}} 17$-cell-mediated autoimmunity to environmental toxins. Nature 453, 106-109 (2008).

68. Cortese, A. et al. Air pollution as a contributor to the inflammatory activity of multiple sclerosis. J. Neuroinflammation 17, 334 (2020).

69. Diaz-Sanchez, D., Dotson, A. R., Takenaka, H. \& Saxon, A. Diesel exhaust particles induce local IgE production in vivo and alter the pattern of IgE messenger RNA isoforms. J. Clin. Invest. 94, 1417-1425 (1994).

70. Li, H. et al. Particulate matter exposure and stress hormone levels: a randomized, double-blind, crossover trial of air purification. Circulation 136, 618-627 (2017).

71. Arumugam, M. et al. Enterotypes of the human gut microbiome. Nature 473, 174-180 (2011)

72. Henrick, B. M. et al. Bifidobacteria-mediated immune system imprinting early in life. Cell 184, 3884-3898 (2021). This study identified a lack of Bifidobacteria in newborn infants as correlating with system inflammation and immune dysregulation. Supplementation of infants with Bifidobacterium subspecies infantis resulted in a shift in gut cytokine production and peripheral $\mathrm{T}$ cell polarisation.

73. Hagan, T. et al. Antibiotics-driven gut microbiome perturbation alters immunity to vaccines in humans. Cell 178, 1313-1328 (2019).

74. van Nood, E. et al. Duodenal infusion of donor feces for recurrent Clostridium difficile. N. Engl. J. Med. 368, 407-415 (2013).

75. Konturek, P. C. et al. Successful therapy of Clostridium difficile infection with fecal microbiota transplantation. J. Physiol. Pharm. 67, 859-866 (2016).

76. Atarashi, K. et al. Ectopic colonization of oral bacteria in the intestine drives $\mathrm{T}_{\mathrm{H}} 1$ cell induction and inflammation. Science 358, 359-365 (2017).

77. Atarashi, K. et al. $\mathrm{T}_{\mathrm{H}} 17$ cell induction by adhesion of microbes to intestinal epithelial cells. Cell 163, 367-380 (2015).

78. Atarashi, $\mathrm{K}$. et al. $\mathrm{T}_{\mathrm{reg}}$ induction by a rationally selected mixture of Clostridia strains from the human microbiota. Nature 500, 232-236 (2013). 
79. Schluter, J. et al. The gut microbiota is associated with immune cell dynamics in humans. Nature 588, 303-307 (2020).

80. Baruch, E. N. et al. Fecal microbiota transplant promotes response in immunotherapy-refractory melanoma patients. Science $\mathbf{3 7 1}$ 602-609 (2021). Microbiome transplantation in people with refractory cancer promoted immunotherapy responses, with associated augmentation of immunological parameters.

81. Panigrahi, P. et al. A randomized synbiotic trial to prevent sepsis among infants in rural India. Nature 548, 407-412 (2017).

82. Hegazy, A. N. et al. Circulating and tissue-resident $\mathrm{CD} 4^{+} \mathrm{T}$ cells with reactivity to intestinal microbiota are abundant in healthy individuals and function is altered during inflammation. Gastroenterology 153, 1320-1337 (2017).

83. Yang, F. et al. Shared B cell memory to coronaviruses and other pathogens varies in human age groups and tissues. Science 372, 738-741 (2021).

84. Schirmer, M. et al. Linking the human gut microbiome to inflammatory cytokine production capacity. Cell 167, 1125-1136 (2016). Parallel studies using microbiome analysis and systems immunology approaches on a healthy cohort identified correlations between microbial taxa and responses to pathogens in vitro.

85. Kamdar, S. et al. Perinatal inflammation influences but does not arrest rapid immune development in preterm babies. Nat. Commun. 11, 1284 (2020).

86. Hansen, L. B. S. et al. A low-gluten diet induces changes in the intestinal microbiome of healthy Danish adults. Nat. Commun. 9, 4630 (2018).

87. Byrd, A. L. et al. Gut microbiome stability and dynamics in healthy donors and patients with non-gastrointestinal cancers. J. Exp. Med. 218, e20200606 (2021).

88. Awany, D. et al. Host and microbiome genome-wide association studies: current state and challenges. Front Genet 9, 637 (2018).

89. Tsang, J. S. et al. Global analyses of human immune variation reveal baseline predictors of postvaccination responses. Cell 157, 499-513 (2014)

90. Nakaya, H. I. et al. Systems analysis of immunity to influenza vaccination across multiple years and in diverse populations reveals shared molecular signatures. Immunity 43, 1186-1198 (2015).

91. Kotliarov, Y. et al. Broad immune activation underlies shared set point signatures for vaccine responsiveness in healthy individuals and disease activity in patients with lupus. Nat. Med. 26, 618-629 (2020). This study identifies a shared baseline immune signature that predicts the immune response after influenza vaccination in s healthy population and predicts disease flare inpatients with systemic lupus erythematosus.

92. Furman, D. et al. Apoptosis and other immune biomarkers predict influenza vaccine responsiveness. Mol. Syst. Biol. 9, 659 (2013).

93. HIPC-CHI Signatures Project Team and HIPC-I Consortium. Multicohort analysis reveals baseline transcriptional predictors of influenza vaccination responses. Sci. Immunol. 2, eaal4656 (2017).

94. Obermoser, G. et al. Systems scale interactive exploration reveals quantitative and qualitative differences in response to influenza and pneumococcal vaccines. Immunity 38, 831-844 (2013).

95. Natrajan, M. S. et al. Systems vaccinology for a live attenuated tularemia vaccine reveals unique transcriptional signatures that predict humoral and cellular immune responses. Vaccines 8, 4 (2019).

96. Rechtien, A. et al. Systems vaccinology identifies an early innate immune signature as a correlate of antibody responses to the ebola vaccine rVSV-ZEBOV. Cell Rep. 20, 2251-2261 (2017).

97. Athale, S. et al. Influenza vaccines differentially regulate the interferon response in human dendritic cell subsets. Sci. Transl. Med. 9, eaaf9194 (2017).

98. Stebegg, M. et al. Rejuvenating conventional dendritic cells and T follicular helper cell formation after vaccination. eLife 9, e52473 (2020).

99. Fourati, S. et al. Pre-vaccination inflammation and B-cell signalling predict age-related hyporesponse to hepatitis B vaccination. Nat. Commun. 7, 10369 (2016).

100. Hung, I. F. et al. Topical imiquimod before intradermal trivalent influenza vaccine for protection against heterologous non-vaccine and antigenically drifted viruses: a single-centre, double-blind, randomised, controlled phase 2b/3 trial. Lancet Infect. Dis. 16, 209-218 (2016).

101. Hung, I. F. et al. Immunogenicity of intradermal trivalent influenza vaccine with topical imiquimod: a double blind randomized controlled trial. Clin. Infect. Dis. 59, 1246-1255 (2014).

102. Grifoni, A. et al. Targets of T cell responses to SARS-CoV-2 coronavirus in humans with COVID-19 disease and unexposed individuals. Cell 181, 1489-1501 e1415 (2020).

103. Low, J. S. et al. Clonal analysis of immunodominance and cross-reactivity of the CD4 T cell response to SARS-CoV-2. Science 372, 1336-1341 (2021).

104. Arunachalam, P. S. et al. Systems biological assessment of immunity to mild versus severe COVID-19 infection in humans. Science 369, 1210-1220 (2020).

105. Hadjadj, J. et al. Impaired type I interferon activity and inflammatory responses in severe COVID-19 patients. Science 369, 718-724 (2020).
106. Penttila, P. A. et al. High dimensional profiling identifies specific immune types along the recovery trajectories of critically ill COVID19 patients. Cell. Mol. Life Sci. 78, 3987-4002 (2021).

107. Vanderbeke, L. et al. Monocyte-driven atypical cytokine storm and aberrant neutrophil activation as key mediators of COVID-19 disease severity. Nat. Commun. 12, 4117 (2021)

108. Ren, X. et al. COVID-19 immune features revealed by a large-scale single-cell transcriptome atlas. Cell 184, 1895-1913 e1819 (2021).

109. Liu, C. et al. Time-resolved systems immunology reveals a late juncture linked to fatal COVID-19. Cell 184, 1836-1857 e1822 (2021).

110. Bergamaschi, L. et al. Longitudinal analysis reveals that delayed bystander $\mathrm{CD}^{+} \mathrm{T}$ cell activation and early immune pathology distinguish severe COVID-19 from mild disease. Immunity 54, 1257-1275 e1258 (2021). This study performed serial immune profiling on 207 SARS-CoV-2-infected individuals, finding an early robust bystander $\mathrm{CD}^{+} \mathrm{T}$ cell response was protective against severe disease, while systemic inflammation was predictive of poor clinical outcomes.

111. Zheng, H. et al. Multi-cohort analysis of host immune response identifies conserved protective and detrimental modules associated with severity across viruses. Immunity 54, 753-768 e755 (2021).

112. de Jong, S. E. et al. Systems analysis and controlled malaria infection in Europeans and Africans elucidate naturally acquired immunity. Nat. Immunol. 22, 654-665 (2021). A rare example of systems immunology during a controlled infection context, with Plasmodium falciparum given to either naive or pre-exposed volunteers. The study identifies both differing baseline and induced responses, with immune variation predicting control of parasitemia.

113. Quach, H. et al. Genetic adaptation and Neandertal admixture shaped the immune system of human populations. Cell 167, 643-656 e617 (2016).

114. Lundtoft, C. et al. Function of multiple sclerosis-protective HLA class I alleles revealed by genome-wide protein-quantitative trait loci mapping of interferon signalling. PLoS Genet. 16, e1009199 (2020).

115. $\mathrm{Li}, \mathrm{Y}$. et al. Inter-individual variability and genetic influences on cytokine responses to bacteria and fungi. Nat. Med. 22, 952-960 (2016).

116. Kwok, A. J., Mentzer, A. \& Knight, J. C. Host genetics and infectious disease: new tools, insights and translational opportunities. Nat. Rev. Genet 22, 137-153 (2021).

117. Severe COVID GWAS Groupet al. Genomewide association study of severe COVID-19 with respiratory failure. N. Engl. J. Med. 383, 1522-1534 (2020).

118. Pairo-Castineira, E. et al. Genetic mechanisms of critical illness in COVID-19. Nature 591, 92-98 (2021).

119. COVID Host-Genetics Initiative. Mapping the human genetic architecture of COVID-19. Nature https://doi.org/10.1038/s41586-021-03767-x (2021).

120. Ansari, M. A. et al. Genome-to-genome analysis highlights the effect of the human innate and adaptive immune systems on the hepatitis $C$ virus. Nat. Genet. 49, 666-673 (2017).

121. Choin, J. et al. Genomic insights into population history and biological adaptation in Oceania. Nature 592, 583-589 (2021).

122. Deschamps, M. et al. Genomic signatures of selective pressures and introgression from archaic Hominins at human innate immunity genes. Am. J. Hum. Genet. 98, 5-21 (2016). A genome-wide study highlights how archaic introgression and selective adaptations have driven the evolution of innate immunity genes.

123. Abi-Rached, L. et al. The shaping of modern human immune systems by multiregional admixture with archaic humans. Science 334, 89-94 (2011).

124. Browning, S. R., Browning, B. L., Zhou, Y., Tucci, S. \& Akey, J. M. Analysis of human sequence data reveals two pulses of archaic Denisovan admixture. Cell 173, 53-61.e59 (2018).

125. Enard, D. \& Petrov, D. A. Evidence that RNA viruses drove adaptive introgression between Neanderthals and modern humans. Cell 175, 360-371.e313 (2018)

126. Rinker, D. C. et al. Neanderthal introgression reintroduced functional ancestral alleles lost in Eurasian populations. Nat. Ecol. Evol. 4 1332-1341 (2020).

127. Zeberg, H. \& Paabo, S. The major genetic risk factor for severe COVID-19 is inherited from Neanderthals. Nature 587, 610-612 (2020).

128. Sams, A. J. et al. Adaptively introgressed Neandertal haplotype at the OAS locus functionally impacts innate immune responses in humans. Genome Biol. 17, 246 (2016).

129. Huffman, J. et al. Alternative splicing of OAS1 alters the risk for severe COVID-19. Preprint at medRxiv https://doi. org/10.1101/2021.03.20.21254005 (2021).

130. Boisson-Dupuis, S. et al. Tuberculosis and impaired IL-23-dependent IFN- $\gamma$ immunity in humans homozygous for a common TYK2 missense variant. Sci. Immunol. 3, eaau8714 (2018).

131. Dendrou, C. A. et al. Resolving TYK2 locus genotype-to-phenotype differences in autoimmunity. Sci. Transl. Med. 8, 363ra149 (2016).

132. Kerner, G. et al. Human ancient DNA analyses reveal the high burden of tuberculosis in Europeans over the last 2,000 years. Am. J. Hum. Genet 108, 517-524 (2021). 
133. Kerner, G. et al. Homozygosity for TYK2 P1104A underlies tuberculosis in about $1 \%$ of patients in a cohort of European ancestry. Proc. Natl Acad. Sci. USA 116, 10430-10434 (2019).

134. Quach, H. \& Quintana-Murci, L. Living in an adaptive world: genomic dissection of the genus Homo and its immune response. J. Exp. Med. 214, 877-894 (2017). This review provides key background on the demographic and adaptive history of Homo sapiens and the admixture of Homo sapiens with archaic, now-extinct hominins, especially in an immune context.

\section{Acknowledgements}

This project was supported by the European Union's Horizon 2020 research and innovation program under grant agreement No 874707 (EXIMIOUS). This work was also supported by the Biotechnology and Biological Sciences Research Council through Institute Strategic Program Grant funding BBS/E/B/000C0427 and BBS/E/B/000C0428. AG is supported by the Research Fund KU Leuven (C24/16/045), the Research Foundation-Flanders (FWO G.0734.15), the Belgian Charcot Foundation, the Queen Elisabeth Medical Foundation and the Horizon2020 'MultipleMS' consortium (grant EU RIA 733161). D. D. acknowledges support from the Laboratoire d'Excellence 'Milieu Intérieur', managed by the Agence Nationale de la Recherche, (ANR-10-LABX-69-01). S. H. B. is supported by KU Leuven BOFZAP start-up grant, VLAIO (Flanders
Innovation \& Entrepreneurship) within the project entitled PRISMA and by a grant from Stichting Alzheimer Onderzoek - Fondation Recherche Maladie Alzheimer (SAO-FMA).

\section{Competing interests}

The authors declare no competing interests.

\section{Additional information}

Correspondence should be addressed to Adrian Liston, Stephanie Humblet-Baron, Darragh Duffy or An Goris.

Peer review information Nature Immunology thanks Mark Davis and the other anonymous, reviewer(s) for their contribution to the peer review of this work. Jamie D. K. Wilson was the primary editor on this article and managed its editorial process and peer review in collaboration with the rest of the editorial team.

Reprints and permissions information is available at www.nature.com/reprints.

Publisher's note Springer Nature remains neutral with regard to jurisdictional claims in published maps and institutional affiliations.

(c) Springer Nature America, Inc. 2021 